\title{
3 Research Suare \\ Plastidial Wax Ester Biosynthesis As A Tool To Synthesize Shorter And More Saturated Wax Esters
}

\section{Katharina Vollheyde}

University of Göttingen: Georg-August-Universitat Gottingen

Ellen Hornung

University of Gottingen: Georg-August-Universitat Gottingen

Cornelia Herrfurth

University of Gottingen: Georg-August-Universitat Gottingen

Till Ischebeck

University of Gottingen: Georg-August-Universitat Gottingen

Ivo Feussner ( $\square$ ifeussn@gwdg.de )

University of Goettingen https://orcid.org/0000-0002-9888-7003

\section{Research}

Keywords: Arabidopsis thaliana, wax ester, metabolic engineering, wax synthase, fatty acid reductase, Marinobacter aquaeolei

Posted Date: August 27th, 2021

DOl: https://doi.org/10.21203/rs.3.rs-840940/v1

License: (c) (i) This work is licensed under a Creative Commons Attribution 4.0 International License. Read Full License

Version of Record: A version of this preprint was published at Biotechnology for Biofuels on December 1st, 2021. See the published version at https://doi.org/10.1186/s13068-021-02062-1. 


\section{Abstract}

Background: Wax esters (WE) are neutral lipids that consist of a fatty alcohol esterified to a fatty acid. WE are valuable feedstocks in industry for producing lubricants, coatings and cosmetics. They can be produced chemically from fossil fuel or plant derived triacylglycerol. As fossil fuel resources are finite, the synthesis of WE in transgenic plants may serve as an alternative source. As chain length and desaturation degree of the alcohol and acyl moieties determine the physicochemical properties of WE and their field of application, a tightly controlled tailor-made WE synthesis in plants is aimed for. Here, we report the generation of ten combinations of WE producing transgenes expressed in Arabidopsis thaliana. In order to study their suitability for WE production in planta, we analyzed WE amount and synthesized WE species in the transgenic plants.

Results: The transgenes consisted of different combinations of a fatty acyl-CoA/ACP reductase (FAR) and two wax synthases/acyl-CoA:diacylglycerol O-acyltransferases (WSD) namely WSD2 and WSD5 from the bacterium Marinobacter aquaeoleoi. We generated constructs with and without plastidial transit peptides to access diverse alcohol and acyl substrate pools within $A$. thaliana. We observed WE formation with plastid and cytosol-localized FAR and WSD in seeds. A comparative WE analysis revealed the production of shorter and more saturated WE by plastid-localized WE biosynthesis compared to cytosolic WE synthesis.

Conclusions: A shift of WE formation into seed plastids is a suitable approach for tailor-made WE production and can be used to synthesize WE mainly derived from mid and long chain saturated and monounsaturated substrates.

\section{Background}

Wax esters (WE) are highly demanded for industrial applications. They are neutral lipids, which are composed of a fatty alcohol esterified to a fatty acid. Chain length and desaturation degree of incorporated alcohol and acyl moieties determine the physicochemical properties of WE (Patel et al., 2001). Giving rise to their various physical and chemical properties, WE have a large range of industrial applications. Among others, they are used in inks, as coatings, for the production of candles, in cosmetics or as lubricants (Rontani, 2010; Wei, 2012). In the past mostly obtained from sperm whale, WE can now be synthesized chemically from fossil fuel or plant derived triacylglycerol (TAG) (Hills, 2003; Karmakar et al., 2017; Vanhercke et al., 2013), or are extracted from seeds of Simmondsia chinensis (Al-Obaidi et al., 2017; Sturtevant et al., 2020).

However, as fossil fuel is a finite source and S. chinensis is not easy to cultivate (Al-Obaidi et al., 2017; Sturtevant et al., 2020), WE production in transgenic plants is discussed as a sustainable and inexpensive solution (Aslan et al., 2015a; Aslan et al., 2015b; Aslan et al., 2014; Heilmann et al., 2012; Ivarson et al., 2017; Iven et al., 2016; Lardizabal et al., 2000; Ruiz-Lopez et al., 2017; Vollheyde et al., 2020; Yu et al., 2018; Zhu et al., 2016). 
To produce WE in plants, two enzymes are needed: Wax synthases (WS) catalyze the formation of WE from fatty alcohols and acyl carrier protein (ACP) or coenzyme A (COA) activated fatty acids. Fatty acylCoA/ACP reductases (FAR) provide fatty alcohols by the reduction of the carboxy group of acyl-CoA/ACP (Hofvander et al., 2011; Reiser and Somerville, 1997; Wahlen et al., 2009; Willis et al., 2011).

Up to now, several combinations of FAR and WS enzymes have been expressed in Arabidopsis thaliana, Camelina sativa, Crambe abyssinica, Brassica carinata, Lepidium campestre and Nicotiana benthamiana (Aslan et al., 2015a; Aslan et al., 2015b; Aslan et al., 2014; Heilmann et al., 2012; Ivarson et al., 2017; Iven et al., 2016; Lardizabal et al., 2000; Ruiz-Lopez et al., 2017; Vollheyde et al., 2020; Yu et al., 2018; Zhu et al., 2016). The studies did not only aim for high WE amounts, but also the synthesis of defined WE species, which are desired for special industrial applications. Especially WE derived from monounsaturated long chain substrates are requested in industry due to excellent lubrication properties (Heilmann et al., 2012). Which and how much WE species are synthesized in planta, depends on one hand on the activities and substrate specificities of the expressed FAR and WS enzymes, on the other hand on the availability of acyl-CoA/ACP substrates: Whereas the expression of mouse FAR and mouse WS led to the formation of WE with mainly polyunsaturated 18 carbon acyl moieties (Heilmann et al., 2012; Iven et al., 2016), enzyme combinations with Marinobacter aquaeolei FAR (MaFAR) and jojoba WS (ScWS), Acinetobacter baylyi WSD1 (AbWSD1) or M. aquaeolei WSD5 (MaWSD5) produced WE with mainly monounsaturated 18 and 20 carbon acyl and alcohol moieties (Iven et al., 2016; Vollheyde et al., 2020; Yu et al., 2018). Interestingly, expression of different enzyme combinations in the high oleic acid mutant fad2 fae1 (Kunst et al., 1992; Okuley et al., 1994) resulted in more than 60 mol\% 18:1/18:1 (alcohol moiety/acyl moiety) WE (Heilmann et al., 2012; Iven et al., 2016; Yu et al., 2018). Likewise, the expression of fatty acid elongases in combination with WE producing enzymes led to the synthesis of longer WE in B. carinata, C. sativa and L. campestre (Ivarson et al., 2017; Zhu et al., 2016). Shorter WE were generated in C. sativa upon co-expression of a 14:0 ACP thioesterase (Ruiz-Lopez et al., 2017).

The above-mentioned studies altered substrate availability through overexpression and knock out/down of fatty acid-synthesizing enzymes. These approaches may have the disadvantage that they (i) interfere with the general fatty acid metabolism of the cell and (ii) additional genes have to be transformed into the WE producing plant. However, as acyl-CoA/ACP biosynthesis gives rise to different acyl-CoA/ACP substrates pools within a plant cell (Hölzl and Dörmann, 2019; Ohlrogge and Jaworski, 1997), we studied here whether a change in the subcellular localization of WE biosynthesis can be used as a tool as well to alter the formation of WE species. The work focused on the comparison of WE species synthesized by plastidial and cytosolic localized WS and FAR enzymes. Due to their lack of transmembrane domains affecting subcellular localization, we expressed combinations of the bacterial M. aquaeolei MaFAR, MaWSD2 and MaWSD5 enzymes in $A$. thaliana with and without plastidial transit peptides and analyzed which WE species are synthesized in the different cellular compartments.

\section{Results}

Generation of transgenic A. thaliana plants expressing combinations of MaFAR, MaWSD2 and MaWSD5 
To compare, which WE species are synthesized from the plastidial in contrast to cytosolic fatty acid pool, we generated transgenic $A$. thaliana plants expressing different combinations of WE forming enzymes with and without plastidial transit peptides. We chose to express FAR and WS enzymes from the bacterium M. aquaeolei (MaFAR (Hofvander et al., 2011; Wahlen et al., 2009), MaWSD2 (Barney et al., 2012; Villa et al., 2014; Vollheyde et al., 2020), MaWSD5 (Knutson et al., 2017; Vollheyde et al., 2020)). Previous studies could show that cytosolic expression of combinations of these enzymes resulted in WE production in planta (Aslan et al., 2015a; Aslan et al., 2015b; Aslan et al., 2014; Heilmann et al., 2012; Ivarson et al., 2017; Iven et al., 2016; Lardizabal et al., 2000; Ruiz-Lopez et al., 2017; Vollheyde et al., 2020; Yu et al., 2018; Zhu et al., 2016). In addition, the lack of transmembrane domains in these bacterial enzymes avoid interference with the subcellular localization of the proteins.

To compare plastidial and cytosolic WE production, we designed constructs following our recently published analysis of transgenic A. thaliana MaFAR/MaWSD5 plants (Vollheyde et al., 2020) and generated nine additional constructs consisting of MaFAR combined with either MaWSD2 or MaWSD5 with and without plastidial transit peptide (Fig. 1, Table 1). All open reading frames were N-terminally fused with a fluorescence tag (yellow fluorescent protein (YFP) or cyan fluorescent protein (CFP)) and an immunotag (myc or flag tag) to facilitate localization studies and western blot analyses. Similar to the published and analyzed MaFAR/MaWSD5 (Bcon::YFP-myc-MaFAR/gly::CFP-flag-MaWSD5) construct (Vollheyde et al., 2020), a construct was created, expressing YFP- and myc-tagged MaFAR under the control of the seed specific ß-conglycinin promoter and CPF- and flag-tagged MaWSD2 under the control of seed specific glycinin promoter (ßcon::YFP-myc-MaFAR/gly::CFP-flag-MaWSD2, from here on referred to as MaFAR/MaWSD2). In addition to these cytosolic enzyme combinations, another set of constructs was made by fusing the three enzymes with an N-terminal plastidial transit peptide (Lee et al., 2002) to direct WE biosynthesis from the cytosol to plastids, the location of de novo fatty acid biosynthesis (cIMaFAR/cIMaWSD2, cIMaFAR/cIMaWSD5). In order to confirm plastidic localization, two constructs were made aiming for a plastidial localization controlled by the $35 \mathrm{~S}$ promoter (35S::clMaFAR/35S:::cIMaWSD2, 35S::clMaFAR/35S:::cIMaWSD5). To analyze a possible contribution of a plastidic fatty alcohol pool in WE production, only a plastid-localized WSD was expressed by four more constructs (gly::clMaWSD2, gly::clMaWSD5, 35S::clMaWSD2, 35S::clMaWSD5).

Transformed $A$. thaliana Col-0 plants were first screened for independent transgenic T1 plants by herbicide treatment with glufosinate and 40 to 100 independent plants were obtained for each construct (Table 1). Despite of two transformation events and screening of a large number of seedlings, only 18 transgenic T1 plants were obtained for MaFAR/MaWSD2. For each construct, ca. 20 independent lines were screened subsequently for high WE content in T2 seeds by WE extraction and thin layer chromatography (TLC) (Iven et al., 2013). For MaFAR/MaWSD5, ten new lines were screened in addition to the ones published by Vollheyde et al. (2020). In case of MaFAR/MaWSD2, cIMaFAR/cIMaWSD2, MaFAR/MaWSD 5 and cIMaFAR/cIMaWSD 5 plants, $50 \%$ to $80 \%$ of the screened lines showed WE formation in T2 seeds. No WE formation was detected in T2 seeds of lines expressing plastidial localized enzymes under the control of the 35S promoter or expressing the plastidial localized MaWSD2 or MaWSD 5 alone. As expression under the control of the 35 S promoter should lead to expression of WE 
synthesizing enzymes in leaves as well, leaves of $\mathrm{T} 1$ plants expressing the respective constructs were screened for WE formation, too. However, no WE formation was detected by TLC analysis.

To examine, whether plastidial localization of proteins was successful with the used transit peptide, 35S:::IMFAR/35S::cIMaWSD2 and 35S::cIMFAR/35S::cIMaWSD5 T2 seedlings were analyzed by confocal microscopy making use of the enzymes' YFP and CFP tags (Fig. 1). Additional file 1 depicts CFP and YFP fluorescence overlay with chlorophyll autofluorescence, confirming plastidial localization of expressed cIMaFAR and cIMaWSD5. No CFP signal was obtained for 35S::cIMFAR/35S::.cIMaWSD2, since only one line was available for analysis.

As significant WE amounts were obtained in T2 seeds of plants expressing the four constructs MaFAR/MaWSD2, cIMaFAR/cIMaWSD2, MaFAR/MaWSD5 and cIMaFAR/cIMaWSD5, three independent lines per construct with high WE levels were chosen for further analyses regarding protein expression, WE content and generated WE species (Table 1). For a comparison with results published for MaFAR/MaWSD5, three additional lines were analyzed in detail as the five already published ones were only analyzed by nanoelectrospray ionization tandem mass spectrometry (nanoESI-MS/MS) (Vollheyde et al., 2020). 
Overview of generated and analyzed transgenic $A$. thaliana plants.

$\begin{array}{ll}\begin{array}{l}\text { Construct expressed (short } \\ \text { name) }\end{array} & \begin{array}{l}\text { Number of } \\ \text { independent } \\ \text { lines after }\end{array} \\ \text { (Bcon/gly: seed specific } & \text { herbicide } \\ \text { promoters, 35S: 35S promoter) } & \text { treatment }\end{array}$

Construct expressed (short

(ßcon/gly: seed specific

promoters, 35S: 35 S promoter)

\section{Number of screened heterozygous lines by TLC (number of lines with increased WE amounts)}

Plant lines used for further analysis ('confocal microscopy

‡western blot

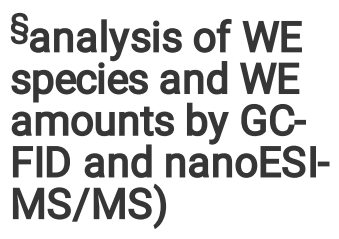

ßcon::YFP-myc-MaFAR 18

T2 seeds: 17 (9)

‡\&lines $2,6,17$

gly::CFP-flag-MaWSD2

(MaFAR/MaWSD2)

Bcon::cl-YFP-myc-MaFAR

85

T2 seeds: 36 (22)

‡S|ines 11, 28, 35

gly::cl-CFP-flag-MaWSD2

(cIMaFAR/cIMaWSD2)

ßcon::YFP-myc-MaFAR

52

T2 seeds: 19 (16)

‡S|ines $11,12,17$

gly::CFP-flag-MaWSD5

[9 (6) lines screened for

(MaFAR/MaWSD5) (Vollheyde et al., 2020), 10 (10) additional lines screened for this

(lines 2, 4, 5, 7, 10 (Vollheyde et al., publication]

Bcon::cl-YFP-myc-MaFAR

43

T2 seeds: 18 (12)

‡Slines 4, 12, 18

gly::cl-CFP-flag-MaWSD 5

(clMaFAR/clMaWSD5)

35S::cl-YFP-myc-MaFAR

72

T2 seeds: $22(0)$

${ }^{\dagger}$ line 21

35S::cl-CFP-flag-MaWSD2

T1 leaves: 5 (0)

(35S::clMaFAR/35S::clMaWSD2)

35S::cl-YFP-myc-MaFAR

56

T2 seeds: $23(0)$

${ }^{+}$lines 18, 19, 23

35S::cl-CFP-flag-MaWSD 5

T1 leaves: 9 (0)

(35S::clMaFAR/35S::clMaWSD5)

gly::cl-CFP-flag-MaWSD2 100

T2 seeds: $21(0)$

gly::cl-CFP-flag-MaWSD5

95

T2 seeds: 21 (0) 


\begin{tabular}{|c|c|c|c|}
\hline $\begin{array}{l}\text { Construct expressed (short } \\
\text { name) } \\
\text { (ßcon/gly: seed specific } \\
\text { promoters, 35S: 35S promoter) }\end{array}$ & $\begin{array}{l}\text { Number of } \\
\text { independent } \\
\text { lines after } \\
\text { herbicide } \\
\text { treatment }\end{array}$ & $\begin{array}{l}\text { Number of screened } \\
\text { heterozygous lines by TLC } \\
\text { (number of lines with } \\
\text { increased WE amounts) }\end{array}$ & $\begin{array}{l}\text { Plant lines used } \\
\text { for further analysis } \\
\text { ('confocal } \\
\text { microscopy } \\
{ }^{+} \text {western blot } \\
\text { Sanalysis of WE } \\
\text { species and WE } \\
\text { amounts by GC- } \\
\text { FID and nanoES- } \\
\text { MS/MS) }\end{array}$ \\
\hline 35S::cl-CFP-flag-MaWSD2 & 50 & $\begin{array}{l}\text { T2 seeds: } 12(0) \\
\text { T1 leaves: } 5(0)\end{array}$ & \\
\hline 35S::cl-CFP-flag-MaWSD5 & 91 & $\begin{array}{l}\text { T2 seeds: } 21(0) \\
\text { T1 leaves: } 38(0)\end{array}$ & \\
\hline
\end{tabular}

\section{MaFAR, MaWSD2 and MaWSD5 protein levels are different in seeds}

Making use of their YFP-myc and CFP-flag tags, protein levels were investigated by western blot analysis in protein extracts of MaFAR/MaWSD2, cIMaFAR/cIMaWSD2, MaFAR/MaWSD 5 and

cIMaFAR/cIMaWSD5 dry T2 seeds. A detection of MaFAR was achieved via an anti-myc IgG antibody and a detection of MaWSD2 as well as MaWSD5 was done via an anti-flag IgG antibody. In addition to that, anti-green fluorescent protein (GFP) IgG antibody was used to monitor all three proteins via their YFP and CFP labels. MaFAR protein was detected in cIMaFAR/cIMaWSD2 and clMaFAR/cIMaWSD5 constructs (Fig. 2, Additional file 2). Despite a weak signal in MaFAR/MaWSD5 line 17, no signal corresponding to MaFAR protein was observed in MaFAR/MaWSD2 and MaFAR/MaWSD5 seeds. A signal corresponding to MaWSD5 protein was obtained in all MaFAR/MaWSD5 and cIMaFAR/cIMaWSD5 lines. In contrast to that, MaWSD2 protein was only detected in the cIMaFAR/cIMaWSD2 lines and as a weak signal in MaFAR/MaWSD2 line 17.

\section{Plastidial WE synthesis leads to a shift in WE length and desaturation degree}

In order to determine WE amount and species generated by the four constructs (MaFAR/MaWSD2, cIMaFAR/cIMaWSD2, MaFAR/MaWSD5 and cIMaFAR/cIMaWSD5), T2 seeds were analyzed by gas chromatography coupled to flame ionization detection (GC-FID) and nanoESI-MS/MS. GC-FID analysis revealed WE contents between 12 and $22 \mathrm{mg} / \mathrm{g}$ seed as well as TAG contents between 188 and $268 \mathrm{mg} / \mathrm{g}$ seed (Fig. 3, Additional files 3 and 4). Although differences in WE levels were not significant between the different constructs, seeds expressing cIMaFAR/CIMaWSD2 contained on average $\sim 50 \%$ less WE than MaFAR/MaWSD2 seeds. In cIMaFAR/cIMaWSD 5 seeds, the averaged WE content was $~ 60 \%$ of the WE amount of MaFAR/MaWSD5 seeds (Fig. 3a). Similar to WE content, no significant difference in TAG content was observed in seeds between the constructs (Fig. 3b). However, MaFAR/MaWSD5 TAG content 
was slightly reduced compared to seeds expressing MaWSD2 and in cIMaFAR/cIMaWSD5 seeds, the TAG content was reduced even more. Figure 3c shows, that MaFAR/MaWSD2 seeds contained on average $8 \%$ WE, which was even higher in an individual line (Additional file 3c). In cIMaFAR/cIMaWSD2 lines, the percentage of WE was $4 \%$. Due to accompanied changes in total TAG amount, WE content in MaFAR/MaWSD 5 and cIMaFAR/cIMaWSD5 seeds accounted to $7 \%$.

In order to analyze whether plastidial localization of WE producing enzymes leads to changes in generated WE species, their fatty acid and fatty alcohol profiles were further investigated by GC-FID (Fig. 4, Additional files 4, 5 and 6). The acyl moiety profile shows only small differences between MaFAR/MaWSD2 and MaFAR/MaWSD5. Both enzyme combinations led to WE consisting mostly of 20:1 (n-9), 18:1 (n-9), 18:2 (n-6) and 16:0 fatty acids (Fig. 4a). For MaFAR/MaWSD5 a significant higher content of $16: 0$ was detected.

Comparing MaFAR/MaWSD2 and cIMaFAR/cIMaWSD2, a plastidial WE biosynthesis led to a significantly reduced incorporation of 18:1 (n-9) accompanied with a higher content of 18:0 and 16:0 acyl moieties (Fig. 4a). Although not significant, a trend towards the reduction of WE with 20:1 (n-9) and 18:2 (n-6) acyl moieties was observed as well. Comparing MaFAR/MaWSD5 and cIMaFAR/cIMaWSD5, a reduced content of 20:1 (n-9) acyl moieties in the plastidial constructs was observed as well and a slight reduction in 16:0 even though these differences were not significant. This was accompanied by an increase in 18:0 and 18:1 (n-9) as well as a significant higher content of 18:1 (n-7) acyl moieties.

The overall chain length and desaturation degree status of acyl moieties reflects the above mentioned trends. For both plastidial constructs a decrease in 20 carbon acyl moieties compared to the corresponding non-plastidial constructs was detected, although this decrease was not significant. Whereas the decrease in 20 carbon chain length species resulted mainly in a significant increase in 16 carbon acyl moieties for cIMaFAR/cIMaWSD2, acyl moieties with 18 carbons chain length increased in CIMaFAR/cIMaWSD5 seed WE (Fig. 4c). The number of double bonds present in acyl moieties did not differ between MaFAR/MaWSD5 and cIMaFAR/cIMaWSD5. In contrast to that, a clear and significant trend towards the incorporation of saturated acyl moieties was observed for cIMaFAR/cIMaWSD2 compared to the corresponding non-plastidial construct. While monounsaturated acyl species were favored by the non-plastidial construct, saturated and monounsaturated acyl moieties were equally distributed in clMaFAR/cIMaWSD2.

Figure $4 \mathrm{~b}$ shows the alcohol moiety profiles of extracted WE. No differences were observed between MaFAR/MaWSD2 and MaFAR/MaWSD5. In both enzyme combinations 20:1 (n-9) and 18:1 (n-9) were the preferred alcohol species incorporated into WE. Comparing the alcohol profiles of plastidial and corresponding non-plastidial constructs, a clear and significant decrease in 20:1 (n-9) alcohol species to almost half of the content was observed, as well as a decrease in 18:1 (n-9). This was accompanied with a large and significant increase in 18:0 alcohol moiety as well as a slight, although not significant, increase in 16:0 in both plastidial constructs. Interestingly, in cIMaFAR/cIMaWSD2 the 18:0 alcohol moiety content was significantly more than in cIMaFAR/cIMaWSD5. 
Figure $4 \mathrm{~d}$ shows the summed up overall chain length and desaturation degree preference for alcohol moieties of WE in the analyzed lines. Whereas alcohol moieties with 20 carbons chain length were preferred over 18 carbons chain length alcohols in non-plastidial constructs, the incorporation of fatty alcohols with 18 carbons chain length was preferred in plastidial constructs. A slight, although not significant, increase in 16 carbon alcohol species was observed in the same combinations as well in comparison to the corresponding non-plastidial constructs. A large shift occurred in the number of double bonds. MaFAR/MaWSD2 and MaFAR/MaWSD 5 preferred monounsaturated alcohol moieties with $~ 70$ mol\%. In both plastidial enzyme combinations, the number of double bonds decreased significantly in alcohol moieties. In cIMaFAR/cIMaWSD 5 saturated and monounsaturated species accounted to equal amounts of $\sim 50 \mathrm{~mol} \%$. In cIMaFAR/cIMaWSD2, the content of saturated and monounsaturated alcohol moieties even inverted compared to MaFAR/MaWSD2 accounting for 70 mol\% saturated moieties in the plastidial construct.

Acyl and alcohol moiety profiles obtained by GC-FID analysis give an overview about the composition of acyl and alcohol species in WE. However, information about individual WE species cannot be obtained by this analysis. Therefore, WE of three independent plant lines per construct were analyzed by nanoESIMS/MS (Fig. 5, Additional file 7). Figure 5 shows the 20 most abundant WE species synthesized by the four analyzed enzyme combinations. As already observed in the GC-FID profiles, seeds expressing either MaFAR/MaWSD2 or MaFAR/MaWSD5 have similar WE compositions. In both constructs, 20:1/18:1 and 20:1/20:1 WE species were the two most abundant WE species, which accounted to $20 \mathrm{~mol} \%$. In cIMaFAR/cIMaWSD2 seeds however, more than 50 mol\% of all WE species contained 18:0 alcohol moieties, which formed the six most abundant WE species. 18:0/18:0 and 18:0/16:0 were the two main WE species in cIMaFAR/cIMaWSD2 seeds, accounting to $30 \mathrm{~mol} \%$. Similar to but not as consistent as in cIMaFAR/cIMaWSD2 seeds, 18:0 alcohol moieties were preferred by clMaFAR/cIMaWSD5 accounting for $\sim 30 \mathrm{~mol} \% .18: 1$ and 18:2 acyl moieties formed the two most abundant WE species in cIMaFAR/cIMaWSD5.

\section{Discussion}

Previous studies suggest that the acyl-CoA pool primarily determines the composition of WE in seeds. Therefore, this study aimed to test whether a shift of WE synthesis from the cytosol to the plastid is an appropriate strategy to change the accessible fatty acid pool and to analyze how this will consequently influence the obtained WE profile. Here, we expressed combinations of $M$. aquaeolei FAR and WSD enzymes in $A$. thaliana with and without plastidial transit peptide and analyzed which WE species are generated by the enzyme combinations in the different cellular compartments. The bacterial enzymes were chosen as they lack transmembrane domains, which might interfere with subcellular protein localization and as they were shown before to be capable to synthesize WE in $A$. thaliana (Aslan et al., 2015a; Aslan et al., 2015b; Aslan et al., 2014; Heilmann et al., 2012; Ivarson et al., 2017; Iven et al., 2016; Lardizabal et al., 2000; Ruiz-Lopez et al., 2017; Vollheyde et al., 2020; Yu et al., 2018; Zhu et al., 2016). 
We detected WE in plants expressing MaFAR and MaWSD combinations in seeds with both cytosolic and plastidial-localized enzymes. Combined GC-FID and nanoESI-MS/MS analyses revealed the expected shift towards shorter and more saturated WE species synthesized by enzymes localized in plastids (Figs. 4 and 5). Seeds of transgenic MaFAR/MaWSD2 and MaFAR/MaWSD5 contained WE with equal amounts of 20:1 (n-9) and 18:1 (n-9) acyl moieties accounting for 20-25 mol\% each, and to a lesser extent with 18:2 (n-6) and 16:0 acyl moieties, accounting for 10-15 mol\% each (Fig. 4). As fatty alcohol moiety, 20:1 (n-9) was favored by both enzyme combinations followed by 18:1 (n-9) (Fig. 4). In contrast to this, seeds expressing the plastidial constructs contained WE with predominantly 18:0 alcohol moieties accounting to 30-50 mol\% (Fig. 4) and the cIMaFAR/cIMaWSD2 seeds accumulated WE with a higher content of 16:0 and 18:0 acyl moieties compared to MaFAR/MaWSD2 seeds (Fig. 4). These results show that a direction of WE biosynthesis to seed plastids can be used as a tool to alter substrate availability for tailor-made WE production. This approach can be used as an alternative to overexpression or downregulation/knocking out of fatty acid modifying enzymes, which was done in the past to alter substrate availability (Heilmann et al., 2012; Ivarson et al., 2017; Iven et al., 2016; Ruiz-Lopez et al., 2017; Yu et al., 2018; Zhu et al., 2016). In favor with this new approach, we did not observe a significant reduction in WE amounts when directing WE biosynthesis from the cytosol to seed plastids (Fig. 3). The here obtained total WE amounts upon plastidial and cytosolic WE formation (12 to $22 \mathrm{mg} / \mathrm{g}$ seed (Fig. 3)) are comparable with WE contents obtained before upon expression of bacterial FAR and WS enzymes in A. thaliana: 4-17 mg/g seed with constructs expressing MaFAR with different bacterial WSD enzymes (Aslan et al., 2015a; Aslan et al., 2015b; Aslan et al., 2014; Heilmann et al., 2012; Ivarson et al., 2017; Iven et al., 2016; Lardizabal et al., 2000; Ruiz-Lopez et al., 2017; Vollheyde et al., 2020; Yu et al., 2018; Zhu et al., 2016) and 4.7-19.8 mg/g seed for MaFAR/MaWSD5 lines (Aslan et al., 2015a; Aslan et al., 2015b; Aslan et al., 2014; Heilmann et al., 2012; Ivarson et al., 2017; Iven et al., 2016; Lardizabal et al., 2000; RuizLopez et al., 2017; Vollheyde et al., 2020; Yu et al., 2018; Zhu et al., 2016).

However, although not significant, a slight trend towards lower WE amounts upon plastidial WE biosynthesis compared to cytosolic WE formation can be seen in the here obtained data (Fig. 3). This might be caused by insufficient WE storage capacities in plastids. Co-expression of structural proteins that coat the WE storing lipid droplets or plastoglobuli in the plastids together with the used FAR/WSD combinations may increase the yield in WE. Another reason for lower WE amounts of seeds expressing plastidial constructs might be a lack of acyl-ACP availability for WE biosynthesis due to efficient fatty acid usage by competing pathways like export or synthesis of other lipids. Aslan et al. (2014) reported that an increase in fatty acid biosynthesis does not lead to an increase in plastidial WE amounts. Coexpression of different plastidial FAR/WS constructs in $N$. benthamiana together with the $A$. thaliana transcription factor AtWRI1, described to induce de novo fatty acid synthesis in plastids (Focks and Benning, 1998; Ma et al., 2013), increased WE formation only for one of the tested enzyme combinations (Aslan et al., 2015a; Aslan et al., 2015b; Aslan et al., 2014; Heilmann et al., 2012; Ivarson et al., 2017; Iven et al., 2016; Lardizabal et al., 2000; Ruiz-Lopez et al., 2017; Vollheyde et al., 2020; Yu et al., 2018; Zhu et al., 2016). In order to increase acyl-ACP availability for plastidial WE biosynthesis, the downregulation of competing pathways might be necessary, but has to be done carefully. Plastidial synthesized fatty acids 
are building blocks for the whole lipid complement of a cell. Hence, a total block of competing acyl-ACP metabolism cannot be achieved. A third explanation for lower WE content in the plastidial lines might be a counter-selection for high expression of the plastidial constructs during the regeneration process as suggested by another study (Aslan et al., 2015b). The authors of this study observed markedly lower WE amounts in stable transformed $N$. benthamiana plants compared to transient transformed once when expressing a fusion construct consisting of a transit peptide, MaFAR and Marinobacter hydrocarbonoclasticus WS2 under the control of the 35S promoter (Aslan et al., 2015a; Aslan et al., 2015b; Aslan et al., 2014; Heilmann et al., 2012; Ivarson et al., 2017; Iven et al., 2016; Lardizabal et al., 2000; Ruiz-Lopez et al., 2017; Vollheyde et al., 2020; Yu et al., 2018; Zhu et al., 2016). They observed that surviving plants showed stunted growth and chlorotic leaves and stems and assumed a counter-selection for high construct expression during the regeneration process.

When expressing plastidial localized enzymes under the control of the $35 \mathrm{~S}$ promoter instead of a seedspecific promoter, we did not observe detectable WE formation in leaves and seeds. This observation may be explained by a counter-selection for high plastidial WE contents as well. Plastidial WE amounts might be more harmful in certain developmental stages than in others. They might be tolerated during later stages of seed development, but might be detrimental during early seedling development. It might be interesting to determine seedling lethality of plants expressing plastid localized WE biosynthesis enzymes under the control of the $35 \mathrm{~S}$ promoter compared to the same proteins under the control of seed specific promoters. However, as screening for transgenic plants was performed in this study through herbicide resistance, a detailed seedling lethality rate was difficult to determine and not analyzed here.

The two most abundant acyl and alcohol moieties in WE from the here analyzed transgenic MaFAR/MaWSD2 and MaFAR/MaWSD5 seeds are 20:1 (n-9) and 18:1 (n-9) moieties (Fig. 4). This preference was also observed in the before analyzed MaFAR/MaWSD5 plants (Aslan et al., 2015a; Aslan et al., 2015b; Aslan et al., 2014; Heilmann et al., 2012; Ivarson et al., 2017; Iven et al., 2016; Lardizabal et al., 2000; Ruiz-Lopez et al., 2017; Vollheyde et al., 2020; Yu et al., 2018; Zhu et al., 2016). In contrast to that, Yu et al. (2018) published acyl and alcohol profiles of seed WE produced by a combination of MaFAR and MaWSD2 with the two most abundant acyl moieties being 18:0 and 18:1 and the two most abundant alcohol moieties being 18:1 and 18:2. It has to be noted, that in the publication by Vollheyde et al. (2020) the same MaFAR/MaWSD 5 constructs were expressed as here, whereas Yu et al. (2018) expressed MaFAR and MaWSD2 without additional YFP-myc/CPF-flag-tags and under the control of the seed specific napin promoter instead of ß-conglycinin and glycinin promoters. The preference for shorter substrates preferred by the MaFAR/MaWSD2 combination described by Yu et al. (2018) compared to the MaFAR/MaWSD2 combination published here may be explained by the different promotors that were chosen to regulate expression of the enzymes. The time of promoter activity during seed development might be different for the napin promoter compared to the ß-conglycinin and the glycinin promoters. Baud et al. (2002) reported that the fatty acid profile changes during the development of $A$. thaliana seeds. While until torpedo stage around $50 \%$ of all seed fatty acids are 16:0 and 18:0 fatty acids, the content of both fatty acids decreases and the amount of 18:3 and 20:1 fatty acids increases during proceeding seed development. This results in different acyl substrate pools available for WE biosynthesis 
over time during seed development. Another explanation for differences in WE acyl and alcohol moiety profiles of MaFAR/MaWSD2 constructs between this study and the work of Yu et al. (2018) might be the presence of N-terminal YFP-myc and CFP-flag fusions in the constructs analyzed here. They might have an influence on the substrate specificities of the proteins. In addition to that, differences in expression levels might be a third explanation, which have not been tested by Yu et al. (2018).

In this study, WE were detected in all analyzed MaFAR/MaWSD2, cIMaFAR/cIMaWSD2, MaFAR/MaWSD5 and cIMaFAR/cIMaWSD5 seeds although western blot analysis did not show in all plant lines detectable protein levels for MaFAR or MaWSD2 under our conditions (Fig. 3 vs. Figure 2). As western blot analysis was performed from dry seeds and WE formation takes place during seed development, differences in protein stability of the expressed enzymes have to be assumed. The detection of WE in all transgenic seeds strongly suggests the presence of all enzymes during seed development.

\section{Conclusions}

A demand for sustainable tailor-made WE production is increasing as fossil fuel resources are finite. Here we report the analysis of transgenic $A$. thaliana plants expressing ten different enzyme combinations of the bacterial MaFAR, MaWSD2 and MaWSD5. A detailed lipid analysis revealed that a direction of WE formation towards plastids in seeds is possible. The availability of acyl-ACP with altered chain length and desaturation degree compared to acyl-CoA present in the cytosolic substrate pool, resulted in the production of shorter and more saturated WE in plastids compared to the cytosol. Consequently, the study presented here shows that a change in subcellular localization of WE biosynthesis is a powerful tool to alter substrate availability for tailor-made WE production in plants.

\section{Methods}

\section{Generation of transgenic $A$. thaliana plants}

Transgenic $A$. thaliana plants were generated according to Vollheyde et al. (2020). Using Gateway technology (Thermo Fischer Scientific) binary transformation vectors were generated for simultaneous transformation of two enzymes as described before (Heilmann et al., 2012).

Using fusion polymerase chain reaction, several constructs were generated from sequence combinations of Escherichia coli codon optimized MaFAR (Accession Number: WP_011785687.1), MaWSD2 (Accession Number: ABM20141.1), MaWSD5 (Accession Number: ABM20482.1), YFP, CFP, myc-tag, flag-tag and a plastidial localization sequence corresponding to an 80 amino acid signal peptide (Lee et al., 2002). As fusion polymerase chain reaction was not successful for generating constructs containing MaWSD2, these constructs were made by classical cloning via an Apal restriction site on the $5^{\prime}$ end of the MaWSD2 sequence. Generated constructs were cloned into desired pENTRY vectors (pENTRYA carrying a 35S promoter, pENTRYB carrying a $\beta$-conglycinin promoter, pENTRYC carrying a glycinin promotor, pENTRYD carrying a 35S promotor) via Sal/ BamHI restriction sites or $\mathrm{Xhol} / \mathrm{Bg} / \mathrm{l}$ restriction sites for MaWSD5 
containing constructs. In total, nine pENTRY vectors were produced. Using Gateway technology (Thermo Fischer Scientific) ten binary vectors were generated from combinations of the destination vector (pCAMBIA33) together with either a combination of pENTRYB, pENTRYC and an empty pENTRYA vector or with a combination of pENTRYA (empty one in case only a WSD will be expressed) and pENTRYD vector. Primer sequences can be found in Additional file 8.

\section{Screening of transgenic $A$. thaliana plants}

Screening of transgenic $A$. thaliana plants by analysis of seed WE was performed as described previously (Vollheyde et al., 2020).

For the screening of transgenic $A$. thaliana plants by leaf WE, three leaves were harvested per plant, pooled and lyophilized. For rapid screening, WE extraction was performed in $2 \mathrm{~mL}$ micro tubes. $500 \mu \mathrm{L}$ methanol were added to lyophilized leaf material and the samples were shaken for 20 min at $4{ }^{\circ} \mathrm{C}$. Afterwards, $1 \mathrm{~mL}$ hexane was added to each sample and samples were shaken for $15 \mathrm{~min}$ at $4{ }^{\circ} \mathrm{C}$. After centrifugation ( $5 \mathrm{~min} 11360 \mathrm{~g}$ ), the upper hexane phase was transferred to a $1.5 \mathrm{~mL}$ microtube. Subsequent to hexane evaporation in a Savant SPD131DDA SpeedVac Concentrator (Thermo Scientific) with a Savant RVT5105 Refrigerated Vapor Trap (Thermo Fischer Scientific), extracted lipids were dissolved in $50 \mu \mathrm{L}$ chloroform and spotted on a TLC silica plate (TLC Silica gel 60, $20 \times 20 \mathrm{~cm}$, Merck Millipore). The TLC plate was developed with hexane/diethyl ether/acetic acid (90:10:1, $v / v / v)$ as running solvent, which yielded best results in separating WE and carotenoids. Bands of neutral lipids were visualized by dipping the plate into a $\mathrm{CuSO}_{4}$ solution $\left(10 \%(\mathrm{~W} / \mathrm{v}) \mathrm{CuSO}_{4}, 6.8 \%(\mathrm{v} / \mathrm{v})\right.$ phosphoric acid) and subsequent heating of the plate to up to $190^{\circ} \mathrm{C}$.

\section{Analysis of WE and TAG by GC-FID}

Lipid extraction, sample preparation and GC-FID analysis of WE and TAG was performed as described previously (Iven et al., 2016).

\section{Analysis of WE species by nanoESI-MS/MS}

WE analysis was performed by nanoESI-MS/MS with a 6500 QTRAP $®$ tandem mass spectrometer (AB Sciex) as previously described (Iven et al., 2013).

\section{Western Blot}

Proteins were extracted from frozen and homogenized seeds. To $4 \mathrm{mg}$ seed material, $100 \mu \mathrm{L}$ freshly prepared extraction buffer (4\% $(w / v)$ SDS, $2 \%(v / v) \beta$-mercaptoethanol, $2 \mathrm{mM}$ phenylmethane sulfonyl fluoride, $0.1 \mathrm{M}$ Tris $\mathrm{pH} 8.5$ ) were added. Samples were immediately vigorously vortexed for at least $2 \mathrm{~min}$. Afterwards, the samples were incubated at $80^{\circ} \mathrm{C}$ for $3 \mathrm{~min}$ and centrifuged $(10 \mathrm{~min}, 20810 \mathrm{~g}$, room temperature). The supernatant was transferred to a new tube and was mixed with $4 x$ Lämmli buffer. For SDS-PAGE and western blot analysis, $10 \mu \mathrm{L}$ of with $4 \mathrm{x}$ Lämmli buffer diluted protein extract was loaded on a SDS gel. For western blot analysis, proteins were detected using an anti-GFP epitope tag antibody 
(diluted 1:5,000, BioLegend), monoclonal anti-c-MYC antibody (1:5,000, Sigma) and monoclonal antiFLAG M2 antibody (1:5,000, Merck) followed by the anti-Mouse IgG (whole molecule)-Alkaline Phosphatase (diluted 1:30,000, Merck). The SDS gel serving as loading control was stained with coomassie.

\section{Microscopy}

Seedlings were grown on MS agar plates containing $1 \%(w / v)$ sucrose for 3 days under long day condition ( $16 \mathrm{~h}$ light, $8 \mathrm{~h}$ darkness, $22^{\circ} \mathrm{C}$ ) subsequent to $2-3$ days of stratification.

Images were recorded using a Zeiss LSM 780 confocal microscope (Carl Zeiss Inc., Jena, Germany). eCFP was excited at $458 \mathrm{~nm}$ and detected at a wavelength of $462-520 \mathrm{~nm}$ imaged using a T80/R20 beam splitter, or at 463-510 nm using a MBS 458 beam splitter; eYFP was excited at $514 \mathrm{~nm}$ and detected at a wavelength of 523-622 nm imaged using a T80/R20 beam splitter, or at 515-551 nm using a MBS $458 / 514$ beam splitter; chlorophyll was excited at $633 \mathrm{~nm}$ and detected at a wavelength of $647-722 \mathrm{~nm}$ imaged using a T80/R20 beam splitter, or at 647-721 nm using a MBS 488/561/633 beam splitter. Images of 35S::cIMaFAR/35S:::cIMaWSD5 lines 18, 19 and 23 (upper image) were recorded with the settings described first. Images of 35S::cIMaFAR/35S::cIMaWSD2 line 21 and 35S::-cIMaFAR/35S:::clMaWSD5 line 23 (lower image) were recorded using the settings described second. Pictures were processed with Image J 1.50i (Schneider et al., 2012).

\section{Abbreviations}

ACP: acyl carrier protein; CFP: cyan fluorescent protein, CoA: coenzyme A; FAR: fatty acyl-CoA/ACP reductase; GC-FID: gas chromatography coupled to flame ionization detection; GFP: green fluorescent protein; nanoESI-MS/MS: nanoelectrospray ionization tandem mass spectrometry; TAG: triacylglycerol; TLC: thin layer chromatography, WE: wax ester; WS: wax synthase, WSD: wax synthases/acylCoA:diacylglycerol $O$-acyltransferases, YFP: yellow fluorescent protein

\section{Declarations}

\section{Ethics approval and consent to participate}

Not applicable

\section{Availability of data and materials}

All data generated or analyzed during this study are included in this published article and its supplementary information files. IF is responsible for distribution of materials presented in this article.

\section{Competing interests}

The authors declare that they have no competing interests. 


\section{Funding}

IF acknowledges funding through the German Research Foundation (DFG, INST 186/822-1).

\section{Authors' contributions}

$\mathrm{KV}$ generated and screened the transgenic plants, performed the GC-FID analysis and data evaluation, prepared the samples for nanoESI-MS/MS measurement, analyzed and evaluated the nanoESI-MS/MS data, performed the western blot analysis and drafted the manuscript. EH participated in cloning the constructs for plant transformation and analyzing the GC-FID data. $\mathrm{CH}$ measured WE species by nanoESIMS/MS. TI recorded the confocal microscopy images. IF designed and supervised the study, analyzed the data and edited the drafted manuscript. All authors read and approved the final manuscript.

\section{Acknowledgements}

The authors are grateful to Prof. Ed Cahoon for providing us with the seed-specific promoters from soybean and Maria Paulat, Andrea Nickel and Susanne Mester for excellent technical assistance. We are also grateful to Dr. Julia Gross for granting access to a confocal microscope and Leonie Witte for her assistance. KV acknowledges the support by the GGNB Program Microbiology and Biochemistry.

\section{References}

1. Al-Obaidi JR, Halabi MF, AlKhalifah NS, Asanar S, Al-Soqeer AA, Attia MF. 2017. A review on plant importance, biotechnological aspects, and cultivation challenges of jojoba plant. Biological Research $50,25$.

2. Aslan S, Hofvander P, Dutta P, Sitbon F, Sun C. 2015a. Transient silencing of the KASIl genes is feasible in Nicotiana benthamiana for metabolic engineering of wax ester composition. Scientific Reports 5, 11213.

3. Aslan S, Hofvander P, Dutta P, Sun C, Sitbon F. 2015b. Increased production of wax esters in transgenic tobacco plants by expression of a fatty acid reductase:wax synthase gene fusion. Transgenic Research 24, 945-953.

4. Aslan S, Sun C, Leonova S, Dutta P, Dörmann P, Domergue F, Stymne S, Hofvander P. 2014. Wax esters of different compositions produced via engineering of leaf chloroplast metabolism in Nicotiana benthamiana. Metabolic Engineering 25, 103-112.

5. Barney BM, Wahlen BD, Garner E, Wei J, Seefeldt LC. 2012. Differences in substrate specificities of five bacterial wax ester synthases. Applied Environmental Microbiology 78, 5734-5745.

6. Baud S, Boutin J-P, Miquel M, Lepiniec L, Rochat C. 2002. An integrated overview of seed development in Arabidopsis thaliana ecotype WS. Plant Physiology and Biochemistry 40, 151-160.

7. Focks N, Benning C. 1998. wrinkled1: A novel, low-seed-oil mutant of Arabidopsis with a deficiency in the seed-specific regulation of carbohydrate metabolism. Plant Physiology 118, 91-101. 
8. Heilmann M, Iven T, Ahmann K, Hornung E, Stymne S, Feussner I. 2012. Production of wax esters in plant seed oils by oleosomal cotargeting of biosynthetic enzymes. Journal of Lipid Research 53, 2153-2161.

9. Hills G. 2003. Industrial use of lipases to produce fatty acid esters. European Journal of Lipid Science and Technology 105, 601-607.

10. Hofvander P, Doan TTP, Hamberg M. 2011. A prokaryotic acyl-CoA reductase performing reduction of fatty acyl-CoA to fatty alcohol. FEBS Letters 585 , 3538-3543.

11. Hölzl G, Dörmann P. 2019. Chloroplast lipids and their biosynthesis. Annual Review of Plant Biology 70, 51-81.

12. Ivarson E, Iven T, Sturtevant D, AhIman A, Cai Y, Chapman K, Feussner I, Zhu L-H. 2017. Production of wax esters in the wild oil species Lepidium campestre. Industrial Crops and Products 108, 535-542.

13. Iven T, Hornung E, Heilmann M, Feussner I. 2016. Synthesis of oleyl oleate wax esters in Arabidopsis thaliana and Camelina sativa seed oil. Plant Biotechnology Journal 14, 252-259.

14. Iven T, Herrfurth C, Hornung E, Heilmann M, Hofvander P, Stymne S, Zhu L-H, Feussner I. 2013. Wax ester profiling of seed oil by nano-electrospray ionization tandem mass spectrometry. Plant Methods 9, 24.

15. Karmakar G, Ghosh P, Sharma BK. 2017. Chemically modifying vegetable oils to prepare green lubricants. Lubricants 5, 44.

16. Knutson CM, Lenneman EM, Barney BM. 2017. Marinobacter as a model organism for wax ester accumulation in bacteria. In: Geiger O, ed. Biogenesis of Fatty Acids, Lipids and Membranes. Cham: Springer International Publishing, 1-22.

17. Kunst L, Taylor DC, Underhill EW. 1992. Fatty acid elongation in developing seeds of Arabidopsis thaliana. Plant Physiology and Biochemistry 30, 425-434.

18. Lardizabal KD, Metz JG, Sakamoto T, Hutton WC, Pollard MR, Lassner MW. 2000. Purification of a Jojoba embryo wax synthase, cloning of its CDNA, and production of high levels of wax in seeds of transgenic Arabidopsis. Plant Physiology 122, 645-656.

19. Lee KH, Kim DH, Lee SW, Kim ZH, Hwang I. 2002. In vivo import experiments in protoplasts reveal the importance of the overall context but not specific amino acid residues of the transit peptide during import into chloroplasts. Molecules \& Cells 14, 388-397.

20. Ma W, Kong Q, Arondel V, Kilaru A, Bates PD, Thrower NA, Benning C, Ohlrogge JB. 2013. WRINKLED1, a ubiquitous regulator in oil accumulating tissues from Arabidopsis embryos to oil palm mesocarp. PLOS ONE 8, e68887.

21. Ohlrogge JB, Jaworski JG. 1997. Regulation of fatty acid synthesis. Annual Review of Plant Physiology and Plant Molecular Biology 48, 109-136.

22. Okuley J, Lightner J, Feldmann K, Yadav N, Lark E, Browse J. 1994. Arabidopsis FAD2 gene encodes the enzyme that is essential for polyunsaturated lipid synthesis. The Plant Cell 6, 147-158. 
23. Patel S, Nelson DR, Gibbs AG. 2001. Chemical and physical analyses of wax ester properties. Journal of Insect Science 1, 4.

24. Reiser S, Somerville C. 1997. Isolation of mutants of Acinetobacter calcoaceticus deficient in wax ester synthesis and complementation of one mutation with a gene encoding a fatty acyl coenzyme $A$ reductase. Journal of Bacteriology 179, 2969-2975.

25. Rontani J-F. 2010. Production of wax esters by bacteria. In: Timmis KN, ed. Handbook of Hydrocarbon and Lipid Microbiology. Berlin, Heidelberg: Springer Berlin Heidelberg, 459-470.

26. Ruiz-Lopez N, Broughton R, Usher S, Salas JJ, Haslam RP, Napier JA, Beaudoin F. 2017. Tailoring the composition of novel wax esters in the seeds of transgenic Camelina sativa through systematic metabolic engineering. Plant Biotechnology Journal 15, 837-849.

27. Schneider CA, Rasband WS, Eliceiri KW. 2012. NIH Image to ImageJ: 25 years of image analysis. Nature Methods 9, 671-675.

28. Sturtevant D, Lu S, Zhou Z-W, Shen Y, Wang S, Song J-M, Zhong J, Burks DJ, Yang Z-Q, Yang Q-Y, Cannon AE, Herrfurth C, Feussner I, Borisjuk L, Munz E, Verbeck GF, Wang X, Azad RK, Singleton B, Dyer JM, Chen L-L, Chapman KD, Guo L. 2020. The genome of jojoba (Simmondsia chinensis): A taxonomically isolated species that directs wax ester accumulation in its seeds. Science Advances 6 , eaay3240.

29. Vanhercke T, Wood CC, Stymne S, Singh SP, Green AG. 2013. Metabolic engineering of plant oils and waxes for use as industrial feedstocks. Plant Biotechnology Journa/ 11, 197-210.

30. Villa JA, Cabezas M, de la Cruz F, Moncalián G. 2014. Use of limited proteolysis and mutagenesis to identify folding domains and sequence motifs critical for wax ester synthase/acyl Coenzyme A:diacylglycerol acyltransferase activity. Applied Environmental Microbiology 80, 1132-1141.

31. Vollheyde K, Yu D, Hornung E, Herrfurth C, Feussner I. 2020. The fifth WS/DGAT enzyme of the bacterium Marinobacter aquaeolei VT8. Lipids 55, 479-949.

32. Wahlen BD, Oswald WS, Seefeldt LC, Barney BM. 2009. Purification, characterization, and potential bacterial wax production role of an NADPH-dependent fatty aldehyde reductase from Marinobacter aquaeolei VT8. Applied Environmental Microbiology 75, 2758-2764.

33. Wei H. 2012. An overview of wax production, requirement and supply in the world market. European Chemical Bulletin 1, 266-268.

34. Willis RM, Wahlen BD, Seefeldt LC, Barney BM. 2011. Characterization of a fatty acyl-CoA reductase from Marinobacter aquaeolei VT8: A bacterial enzyme catalyzing the reduction of fatty acyl-CoA to fatty alcohol. Biochemistry 50, 10550-10558.

35. Yu D, Hornung E, Iven T, Feussner I. 2018. High-level accumulation of oleyl oleate in plant seed oil by abundant supply of oleic acid substrates to efficient wax ester synthesis enzymes. Biotechnology for Biofuels 11, 53.

36. Zhu L-H, Krens F, Smith MA, Li X, Qi W, van Loo EN, Iven T, Feussner I, Nazarenus TJ, Huai D, Taylor DC, Zhou X-R, Green AG, Shockey J, Klasson KT, Mullen RT, Huang B, Dyer JM, Cahoon EB. 2016. 
Dedicated industrial oilseed crops as metabolic engineering platforms for sustainable industrial feedstock production. Scientific Reports 6, 22181.

\section{Figures}
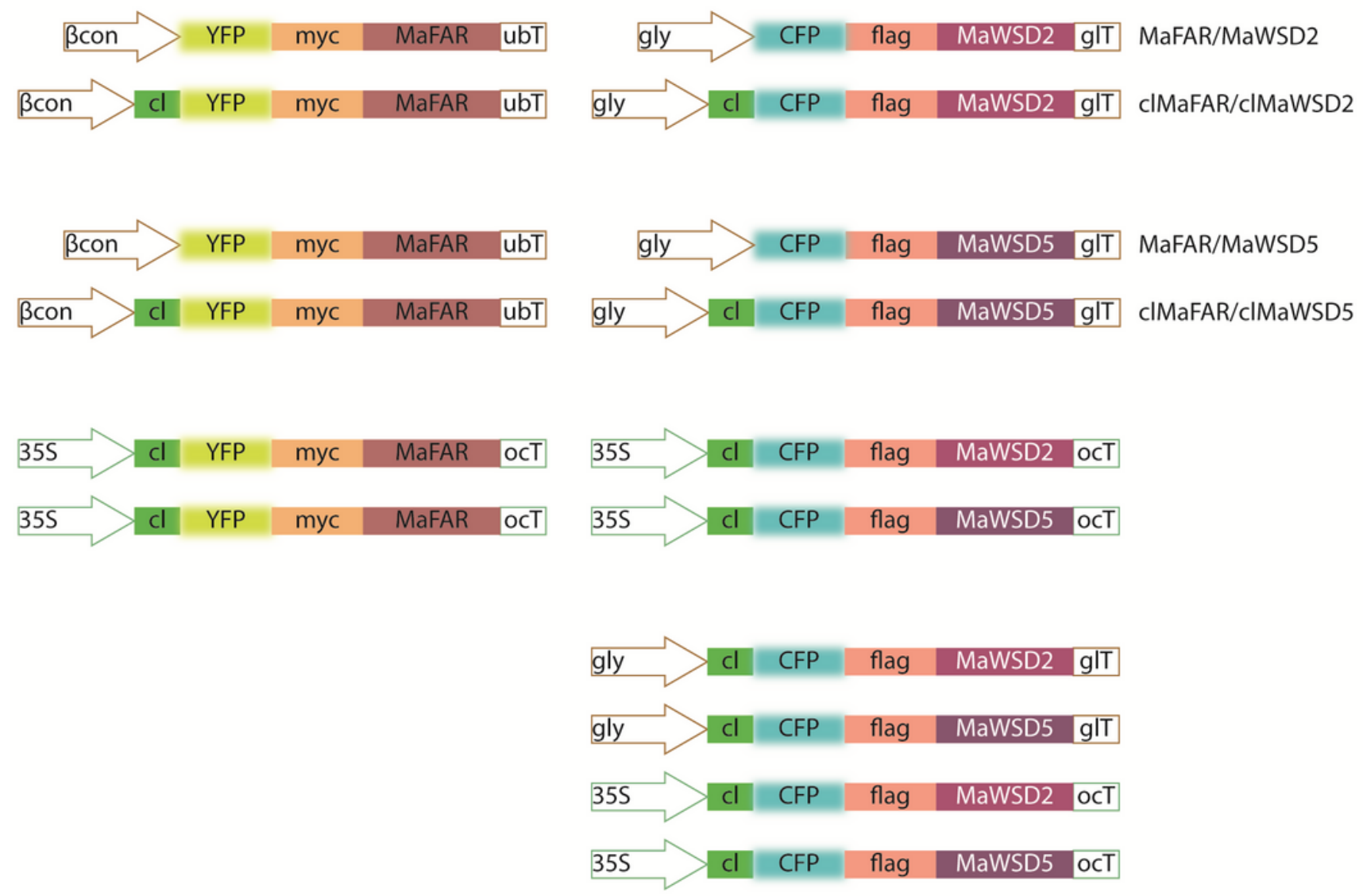

\section{Figure 1}

Constructs generated for A. thaliana transformation. The MaFAR/MaWSD5 construct was published recently (Vollheyde et al., 2020). ßcon: ß-conglycine promoter (Glycine max), seed specific; gly: glycinin promoter (G. max), seed specific; 35S: 35S promoter, ubiquitous promoter; ubT: ubiquitin 3 terminator; glT: glycinin terminator; ocT: octopine synthase terminator. 


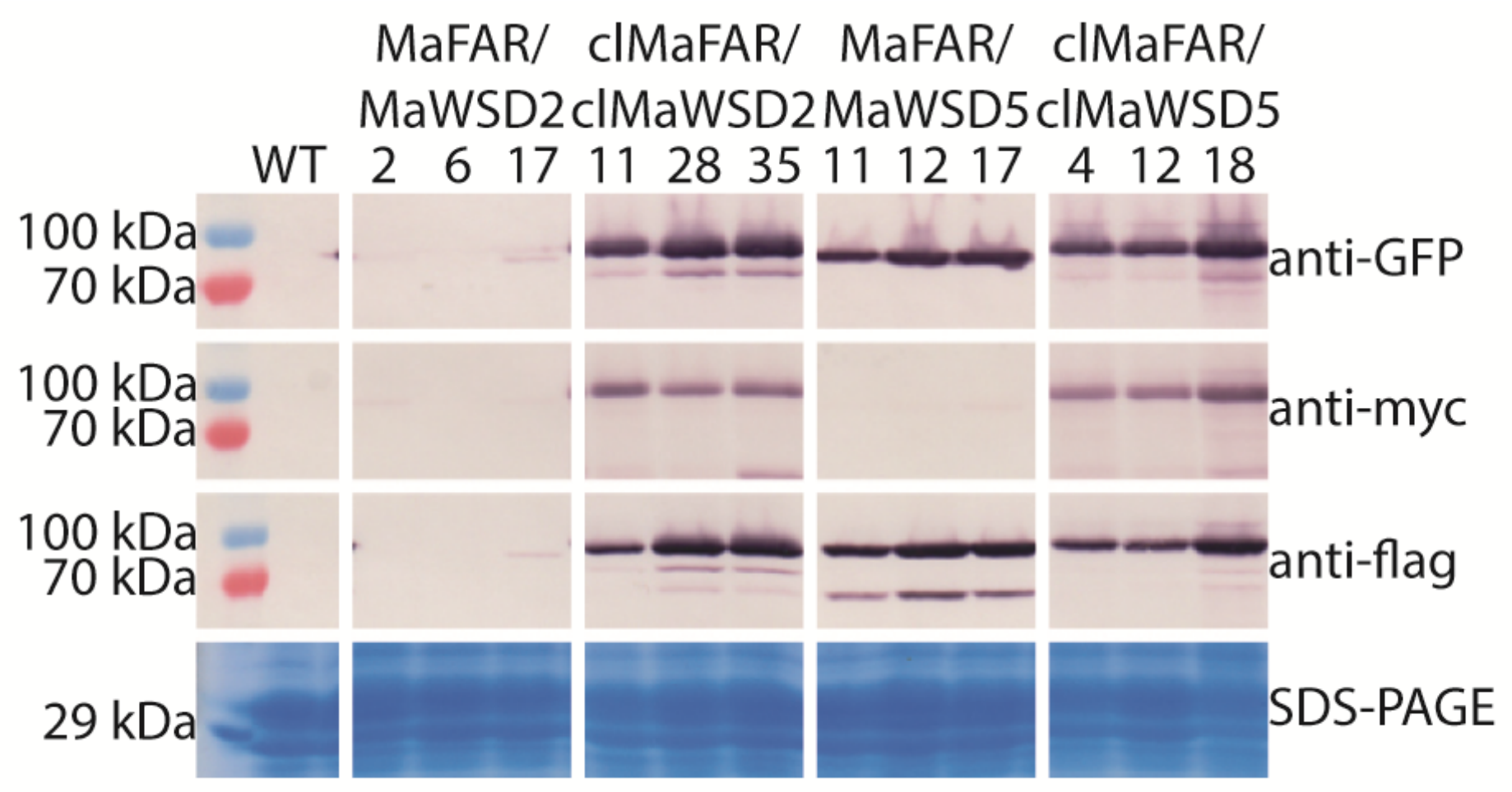

Figure 2

Western blot analysis of MaFAR/MaWSD2, clMaFAR/cIMaWSD2, MaFAR/MaWSD5 and clMaFAR/cIMaWSD5 seeds. Equal amounts of total T2 seed protein extracts were loaded on SDS gels for western blot analyses and SDS-PAGE. Protein detection was achieved by anti-GFP, anti-myc and anti-flag IgG antibodies followed by the anti-Mouse IgG (whole molecule)-Alkaline Phosphatase. The SDS-PAGE gel, serving as loading control, was stained with coomassie. The experiment was performed once analyzing three independent plant lines per construct (MaFAR/MaWSD2: $\beta$ con::YFP-mycMaFAR/gly::CFP-flag-MaWSD2, clMaFAR/clMaWSD2: ßcon::cl-YFP-myc-MaFAR/gly::cl-CFP-flagMaWSD2, MaFAR/MaWSD5: ßcon::YFP-myc-MaFAR/gly::CFP-flag-MaWSD5, clMaFAR/clMaWSD5: Bcon::cl-YFP-myc-MaFAR/gly::cl-CFP-flag-MaWSD5). For images of whole membranes and gel, see Additional file 2. 
(a)

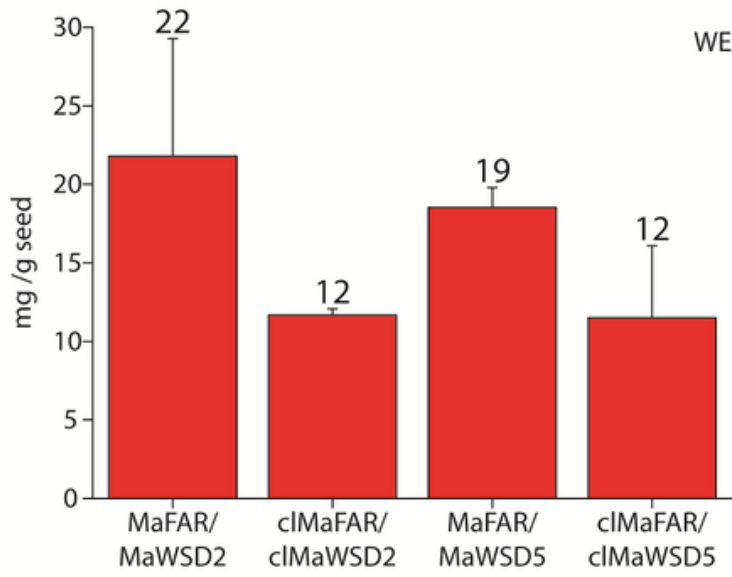

(b)

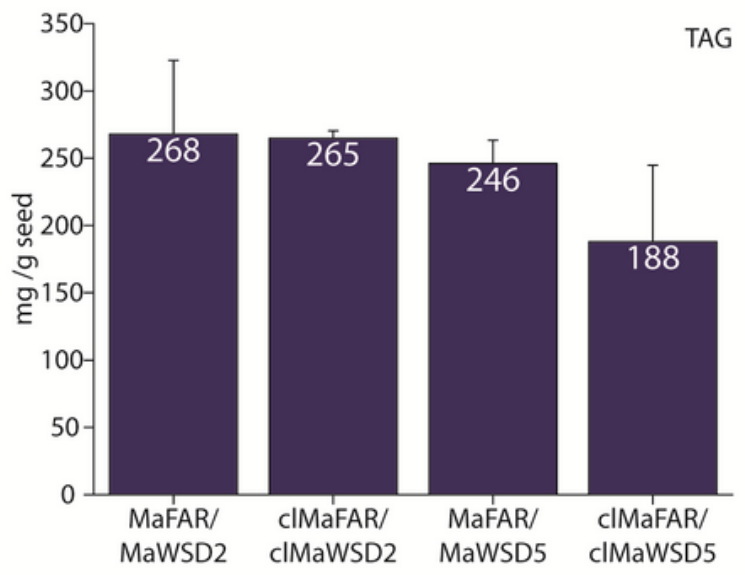

(c)

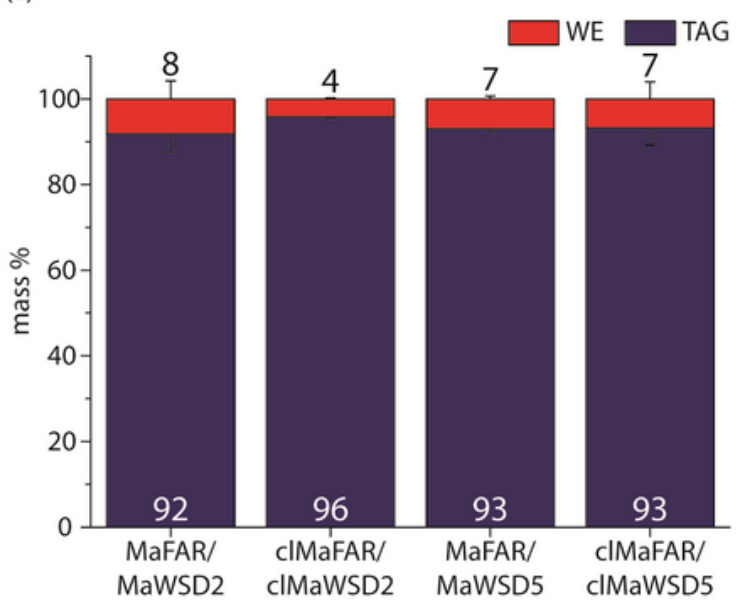

Figure 3

WE and TAG content of MaFAR/MaWSD2, cIMaFAR/cIMaWSD2, MaFAR/MaWSD5 and cIMaFAR/cIMaWSD 5 seeds. Absolute WE (a) and TAG (b) amounts in $\mathrm{mg} / \mathrm{g}$ seed were obtained by GCFID analysis. Both values were used to calculate their relative portion in mass \% (c). Each bar represents the mean of three independent plant lines per construct determined in three extraction replicates (+SD). Analysis of variance (ANOVA) revealed no significant differences in absolute and relative WE and TAG 
contents between the constructs. For the data from each plant line, see Additional file 3. The raw data are provided in Additional file 4.

(a)

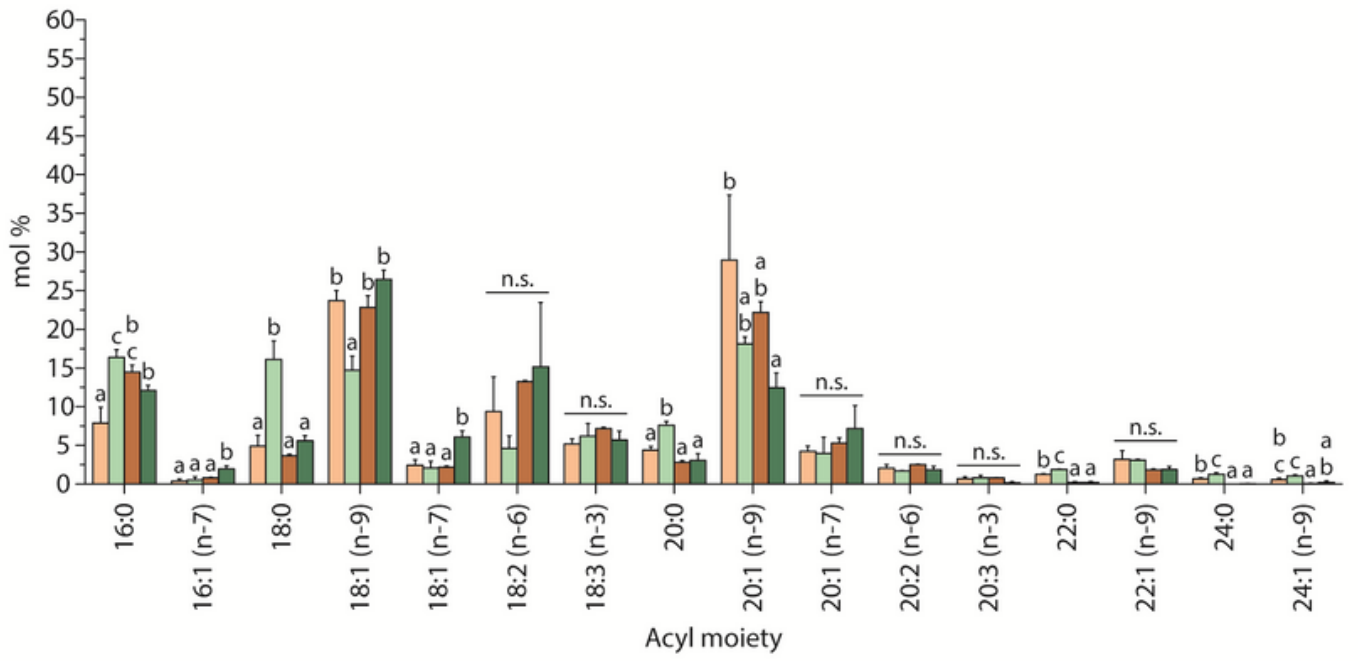

(b)

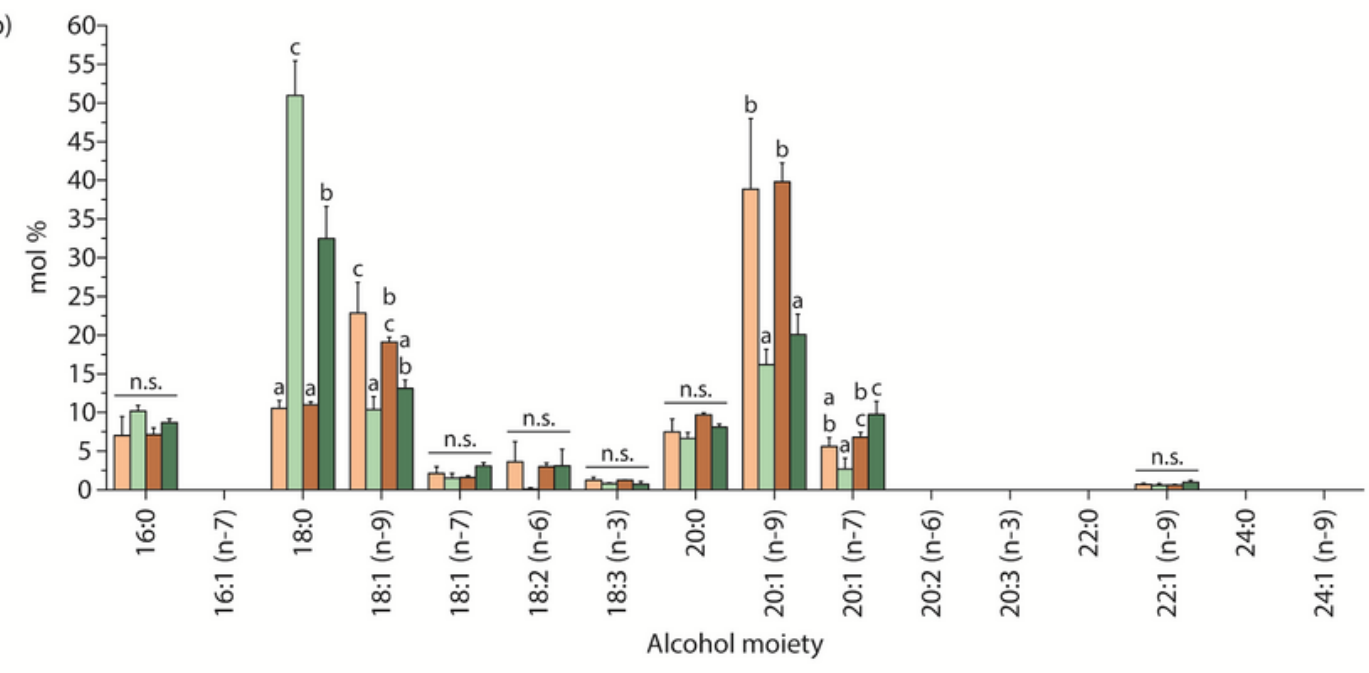

(c)

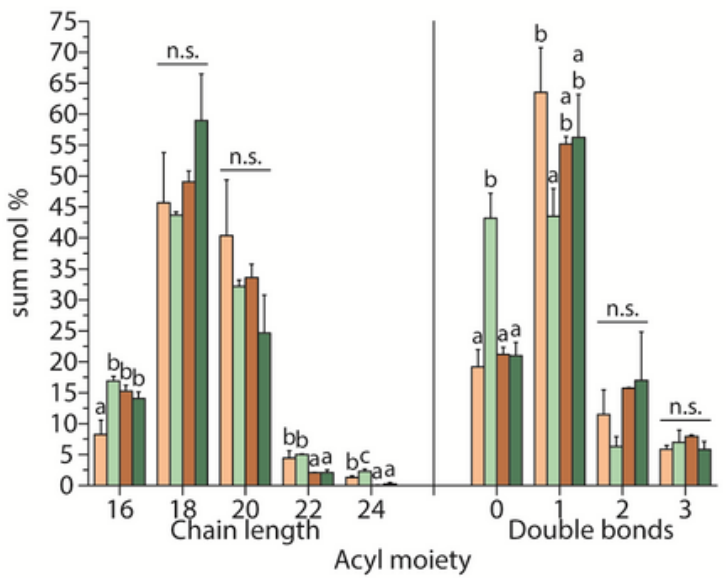

MaFAR/MaWSD2 (d)

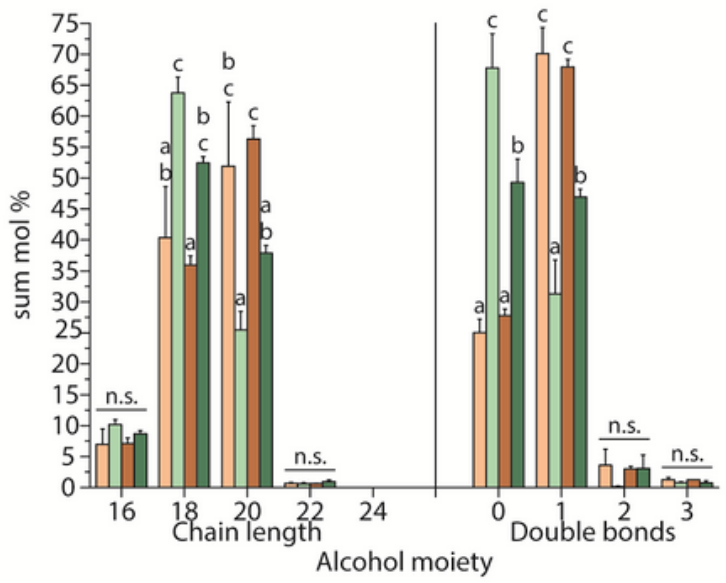

MaFAR/MaWSD5
cIMaFAR/cIMaWSD5

Figure 4

Acyl and alcohol moiety profiles of seed WE from MaFAR/MaWSD2, cIMaFAR/cIMaWSD2, MaFAR/MaWSD5 and cIMaFAR/clMaWSD5. Acyl (a) and alcohol (b) moiety profiles were obtained by GC-FID analysis. Displayed are relative abundances of WE moieties in mol\%. Combined relative 
abundances of acyl (c) and alcohol (d) moieties with similar chain length or desaturation degree were attained by summing up relative abundances of respective moieties. Each bar represents the mean of three independent plant lines per construct determined in three extraction replicates (+SD). ANOVA analysis followed by post-hoc Tukey test was performed separately for each acyl and alcohol moiety as well as each chain length and double bond number (n.s.: not significant). For p-values of the ANOVA analysis, see Additional file 6. For the data from each plant line, see Additional file 5. The raw data are provided in Additional file 4.

(a)

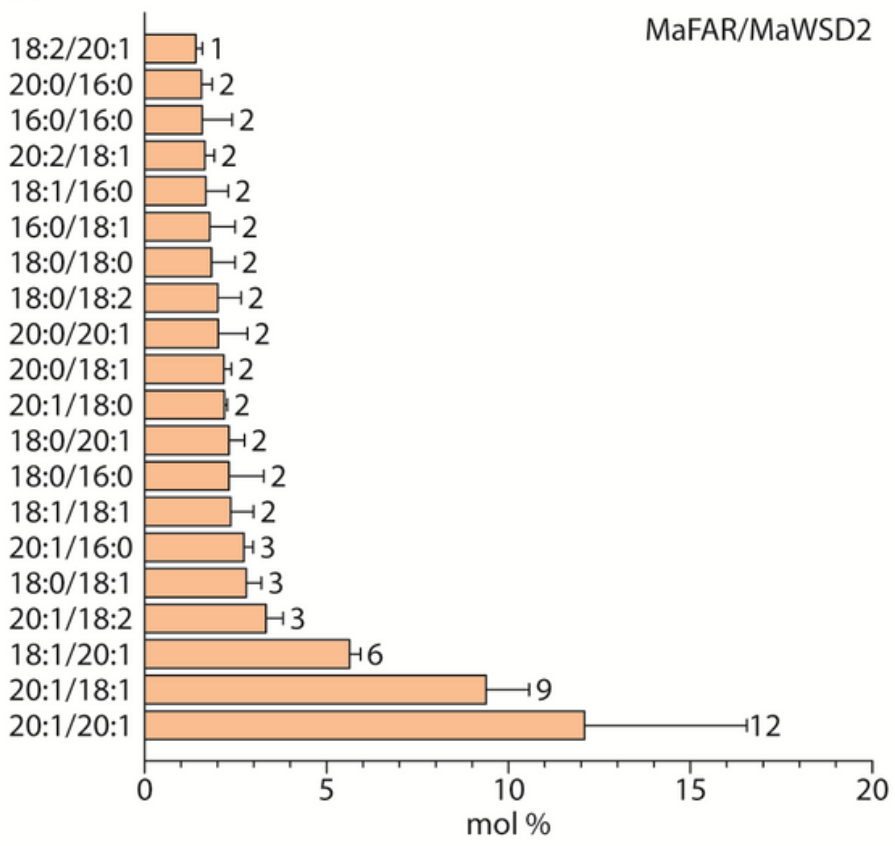

(c)

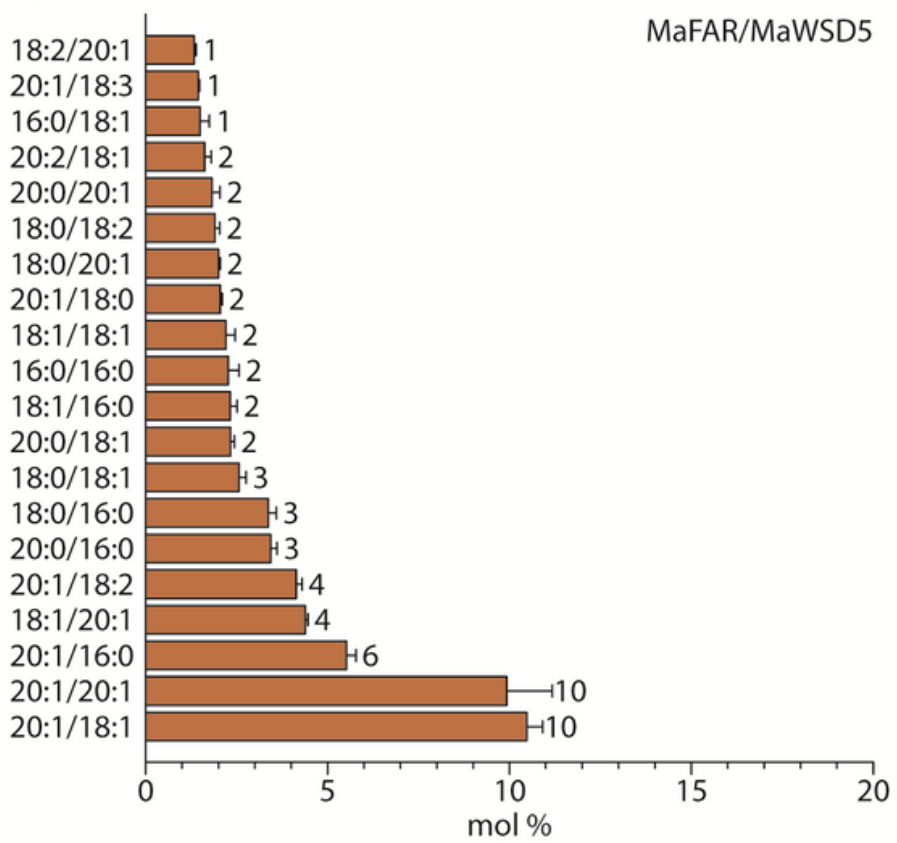

(b)

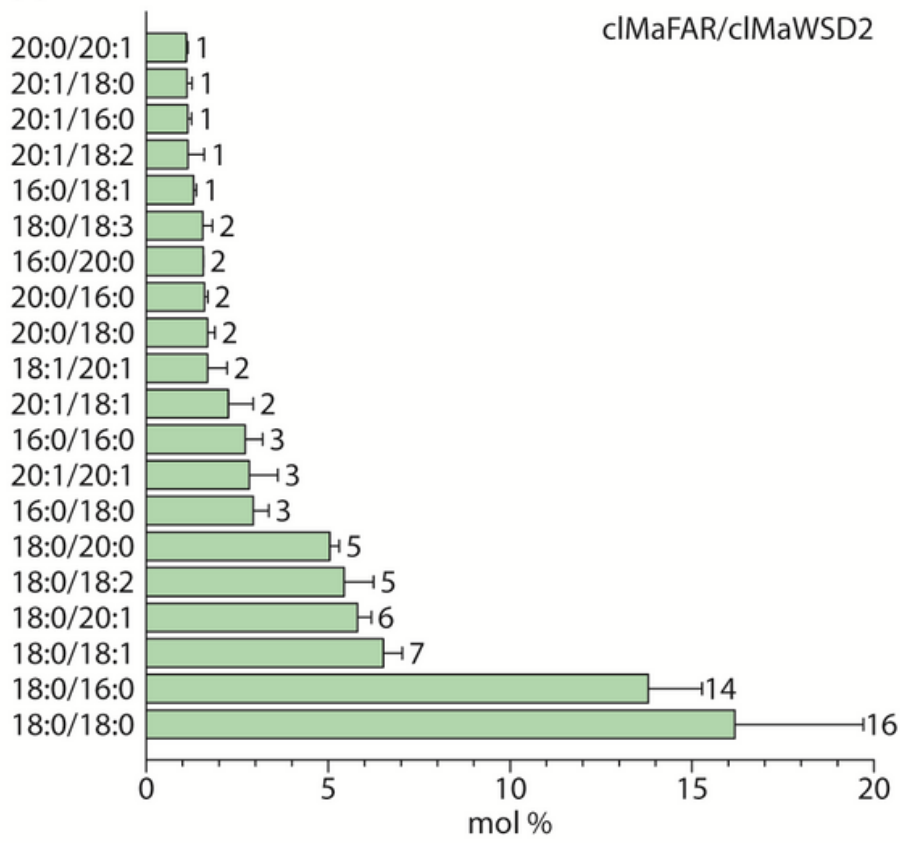

(d)

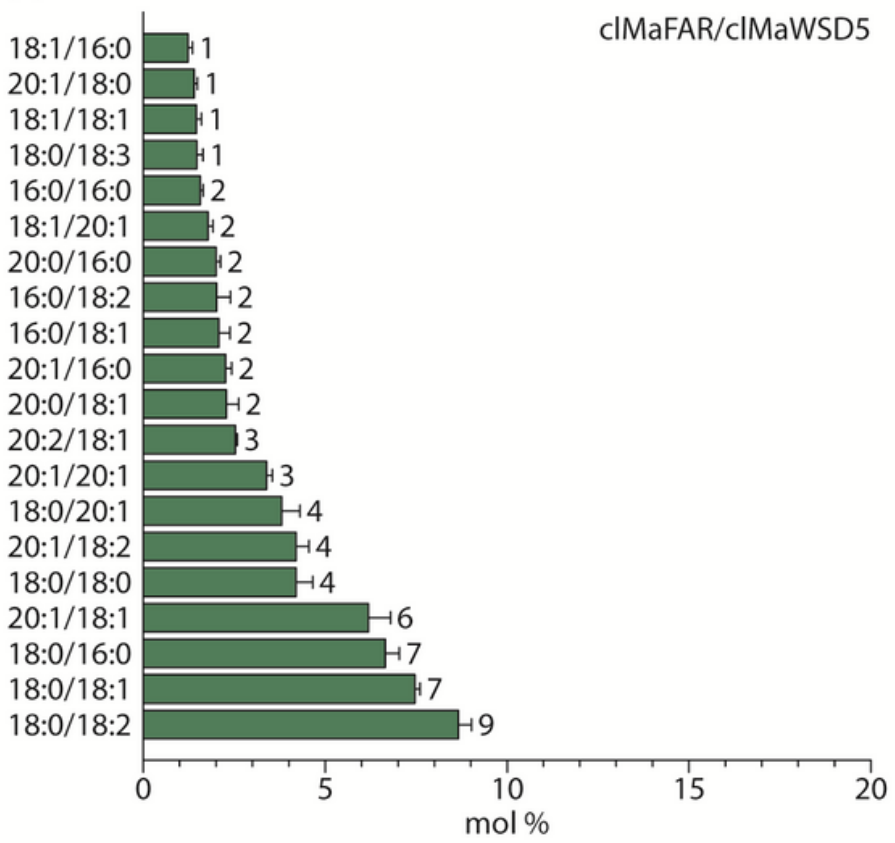

\section{Figure 5}


Analysis of seed WE species in MaFAR/MaWSD2, cIMaFAR/cIMaWSD2, MaFAR/MaWSD5 and cIMaFAR/cIMaWSD5. Molecular WE species were analyzed by nanoESI-MS/MS in MaFAR/MaWSD2 (a), cIMaFAR/cIMaWSD2 (b), MaFAR/MaWSD5 (c) and cIMaFAR/cIMaWSD5 (d) T2 seeds. Displayed are relative abundances of the top 20 WE species (alcohol moiety/acid moiety) of each construct in mol\%. Each bar represents the mean of three independent plant lines per construct determined in three measuring replicates (+SD). The raw data are provided in Additional file 7.

\section{Supplementary Files}

This is a list of supplementary files associated with this preprint. Click to download.

- Additionalfile1.tif

- Additionalfile2.tif

- Additionalfile3.tif

- Additionalfile4.xlsx

- Additionalfile5.tif

- Additionalfile6.docx

- Additionalfile7.xlsx

- Additionalfile8.docx 